\title{
Précipitation dans MgO implanté avec des ions alcalins
}

\author{
M. Treilleux et G. Chassagne \\ Département de Physique des Matériaux (*), \\ Université Claude Bernard Lyon I, \\ 43, bd du 11 novembre 1918, 69621 Villeurbanne, France
}

(Reçu le 20 décembre 1978, accepté le 8 février 1979)

\begin{abstract}
Résumé. - Nous avons observé, par microscopie électronique à transmission, des monocristaux d'oxyde de magnésium implantés avec des ions $\mathrm{Li}^{+}$et $\mathrm{Na}^{+}$et recuits. Ces cristaux présentent, dans la zone d'implantation, une forte densité de dislocations, et les précipités de particules implantées se forment de préférence sur ces dislocations. Lorsque le paramètre cristallin est voisin de celui de la matrice, il y a épitaxie simple avec celle-ci.
\end{abstract}

\begin{abstract}
Magnesium oxide single crystals implanted with $\mathrm{Li}^{+}$and $\mathrm{Na}^{+}$ions and annealed have been observed by transmission electron microscopy. In the implantation zone these crystals exhibit a high density of dislocations, and precipitates of implanted particles nucleate preferably on these dislocations. When the lattice parameter is close to that of the matrix, a simple epitaxial relationship exists.
\end{abstract}

1. Introduction. - Dans un récent article [1], nous nous sommes intéressés aux agrégats de potassium obtenus par recuit de monocristaux d'oxyde de magnésium monocristallin implantés avec des ions $\mathrm{K}^{+}$ de $470 \mathrm{keV}$. L'orientation de ces précipités par rapport à la matrice n'est pas simple; cependant, un accord épitaxique existe puisque les précipités fondus recristallisent dans la même orientation. Dans la présente lettre, nous indiquerons nos premiers résultats concernant d'autres métaux alcalins (lithium et sodium).

Dans des monocristaux d'oxyde de magnésium (provenant de W. C. Spicer L.t.d., St Mary's Winchcombe, Cheltenham Gloucestershire, U.K.) de pureté $4 \mathrm{~N}$, clivés suivant $\langle 100\rangle$, nous avons implanté des ions lithium d'énergie $250 \mathrm{keV}$ et $60 \mathrm{keV}$, et sodium d'énergie $300 \mathrm{keV}$, à l'aide d'un implanteur Danfysik. Le courant de faisceau lors de l'implantation était de $1 \mu \mathrm{A}$ et les doses de l'ordre de $10^{17}$ ions. $\mathrm{cm}^{-2}$. La profondeur moyenne d'implantation $R_{\mathrm{p}}$ est, dans ces conditions, de $6500 \AA$ pour $\mathrm{Li}^{+}(250 \mathrm{keV})$ et de l'ordre de $3000 \AA$ pour $\mathrm{Li}^{+}(60 \mathrm{keV})$ et $\mathrm{Na}^{+}(300 \mathrm{keV})$, avec des écarts types respectifs $\Delta R_{\mathrm{p}}$ de $800 \AA\left(\mathrm{Li}^{+}\right.$ $250 \mathrm{keV})$ et de $600 \AA\left(\mathrm{Li}^{+} 60 \mathrm{keV}, \mathrm{Na}^{+} 300 \mathrm{keV}\right)$.

Ces échantillons, après un recuit à $773 \mathrm{~K}$, présentent des bandes d'absorption optique [2], [3] liées à la présence dans la matrice de précipités de particules implantées. Pour des recuits à des températures plus

(*) Associé au C.N.R.S. élevées, l'évolution de ces bandes indique que ces précipités grossissent.

2. Etude des précipités par microscopie électronique à transmission. - 2.1 PRÉPARATION DES LAMES MINCES. - Les lames minces de MgO, implantées et recuites, d'épaisseur $0,3 \mathrm{~mm}$ ont été préparées par amincissement chimique du côté non implanté jusqu'à apparition d'un trou. Ensuite, par bombardement avec des ions argon de $5 \mathrm{keV}$ sous une incidence presque rasante (angle d'incidence $75^{\circ}$ ), quelques milliers d'angströms sont enlevés pour atteindre la couche implantée sans la supprimer.

2.2 Observation. - Quelle que soit la nature de la particule implantée, la zone d'implantation est fortement endommagée. Dans le cas de l'ion le plus léger $\left(\mathrm{Li}^{+}\right)$, le contraste des dommages est bien résolu : la figure 1 montre un réseau de dislocations, ainsi que des boucles de dislocations (A, Fig. 1). Nous avons caractérisé la nature de ces boucles par la méthode proposée par B. Edmonson et G. K. Williamson [4] : ces boucles sont prismatiques, de vecteur de Burgers $\frac{1}{2}\langle 110\rangle$, et de caractère interstitiel. Des boucles de dislocations de même type ont été observées par D. G. Howitt et al. [5] et aussi par G. W. Groves et A. Kelly [6] respectivement dans $\mathrm{MgO}$ irradié aux électrons et aux neutrons. Cela suggère que la forte densité de dislocations observée est due à l'intersection de telles boucles.

De façon générale, quelle que soit la nature de l'ion, la taille des précipités augmente avec la température 


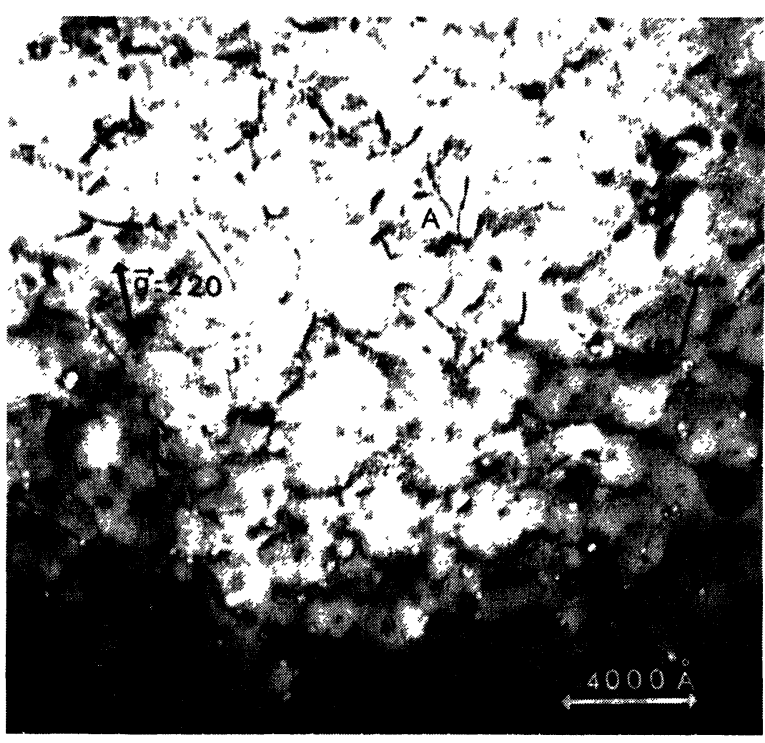

Fig. 1. - MgO implanté avec des ions $\mathrm{Li}^{+}$de $250 \mathrm{keV}$ $\left(10^{17}\right.$ ions. $\left.\mathrm{cm}^{-2}\right)$ et recuit à $1173 \mathrm{~K}$.

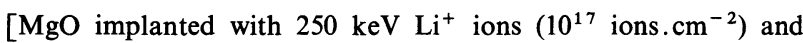
annealed at $1173 \mathrm{~K}$.] de recuit en même temps que leur concentration diminue (Fig. 2). Pour une température de recuit de $1073 \mathrm{~K}$, les plus gros précipités mesurent environ $200 \AA$ et pour $1173 \mathrm{~K}, 500$ à $600 \AA$. La nucléation s'effectue de préférence sur les défauts du cristal : ceci est clairement mis en évidence dans le cas du lithium (Fig. 1), où les plus gros précipités sont observés sur des lignes de dislocations.

2.3 IDENTIFICATION DES PRÉCIPITÉS. - 2.3.1 Cas du lithium. - Après un recuit à $1173 \mathrm{~K}$, les précipités sont suffisamment gros pour que l'on puisse obtenir un diagramme de diffraction (Fig. 3). Les distances interréticulaires sont déterminées à partir de celles de $\mathrm{MgO}$ prises pour référence et permettent, à partir de plusieurs clichés de diffraction, de déterminer la structure (cubique centrée) et le paramètre $(a=3,50 \AA)$ de ces précipités : ce qui correspond au lithium.

Pour des températures de recuit inférieures (1 $073 \mathrm{~K}$ $973 \mathrm{~K}$ ), il n'est pas possible de diffracter sur un seul précipité. Cependant, dans les conditions de Bragg, sur une réflexion (111) de $\mathrm{MgO}$, on observe des franges de moiré à l'intérieur des précipités (Fig. 4). Ces

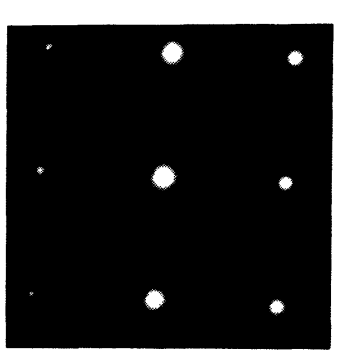

a)

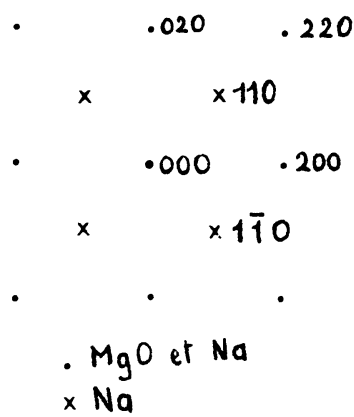

$\times \mathrm{Na}$

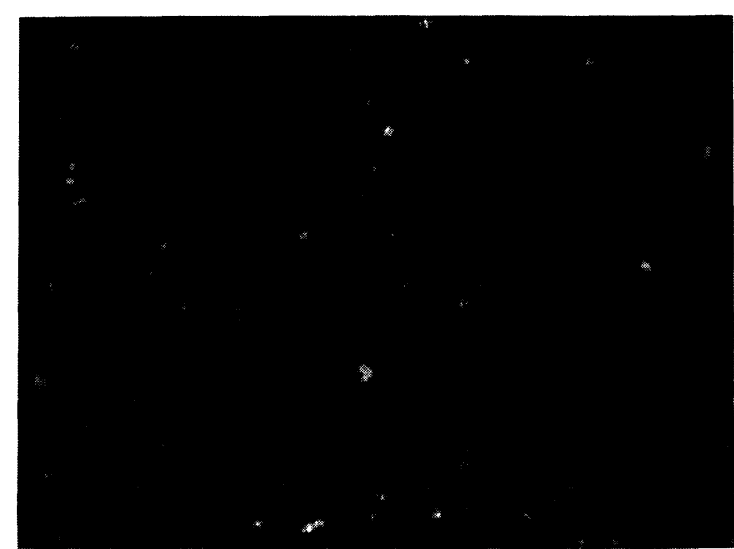

b)

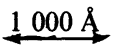

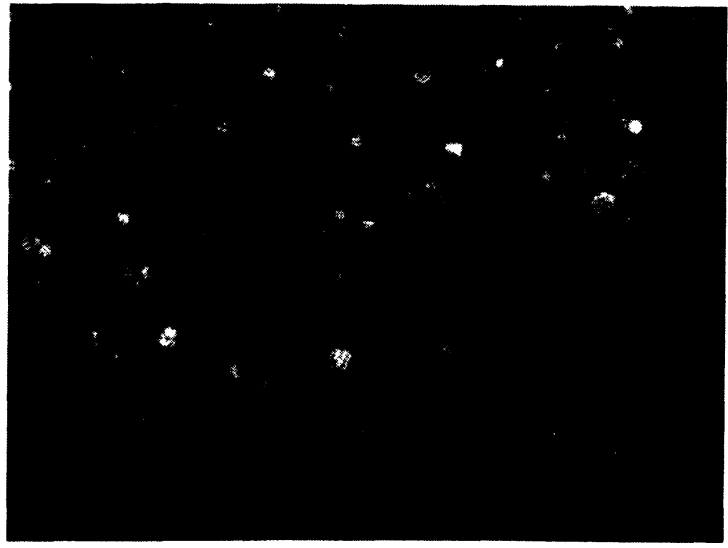

d)

\section{$2000 \AA$}

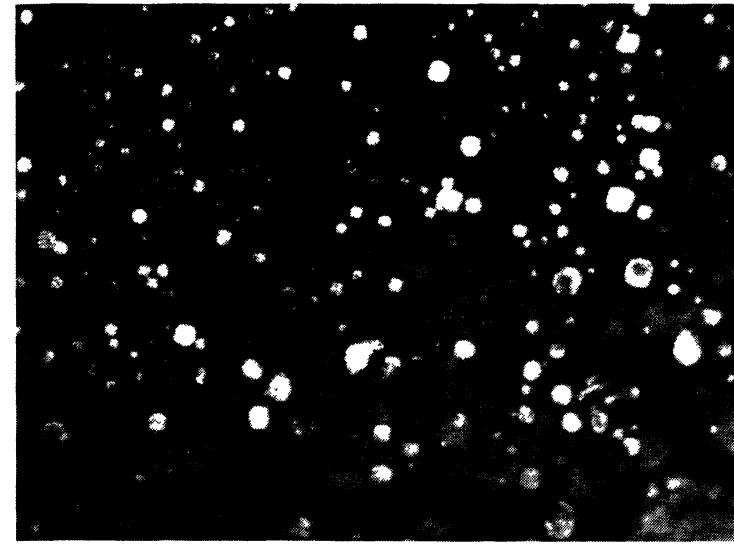

c)

$2000 \AA$

Fig. 2. - MgO implanté avec des ions $\mathrm{Na}^{+}$de $300 \mathrm{keV}\left(10^{17}\right.$ ions. $\left.\mathrm{cm}^{-2}\right)$ et recuit $\left.: a\right)$ diagramme de diffraction ; $\left.b\right)$ recuit $1073 \mathrm{~K}: \mathrm{champ}$ sombre avec $(1 \overline{1} 0) * \mathrm{Na} ; c)$ recuit $1173 \mathrm{~K}:$ champ sombre avec $\left.(110){ }^{*} \mathrm{Na} ; d\right)$ recuit $1173 \mathrm{~K}:$ champ sombre avec $(1 \overline{1} 0){ }^{*} \mathrm{Na}$.

[MgO implanted with $300 \mathrm{keV} \mathrm{Na}{ }^{+}$ions $\left(10^{17}\right.$ ions. $\left.\mathrm{cm}^{-2}\right)$ and annealed $\left.: a\right)$ diffraction pattern; $\left.b\right)$ annealed at $1073 \mathrm{~K}:$ dark field with $(110) * \mathrm{Na} ; c)$ annealed at $1173 \mathrm{~K}$ : dark field with $\left.(110){ }^{*} \mathrm{Na} ; d\right)$ annealed at $1173 \mathrm{~K}:$ dark field with $(1 \overline{10}) * \mathrm{Na}$.] 


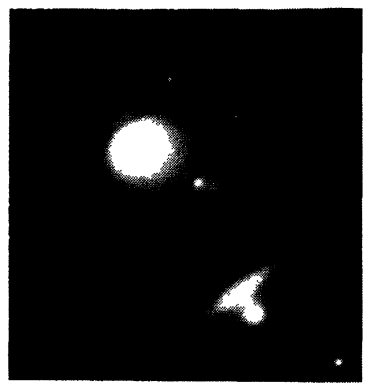

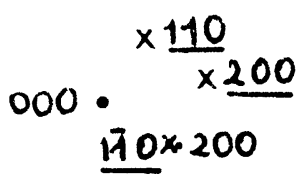

$.2 \overline{2} 0$

$\stackrel{2000 \AA}{\longrightarrow}$

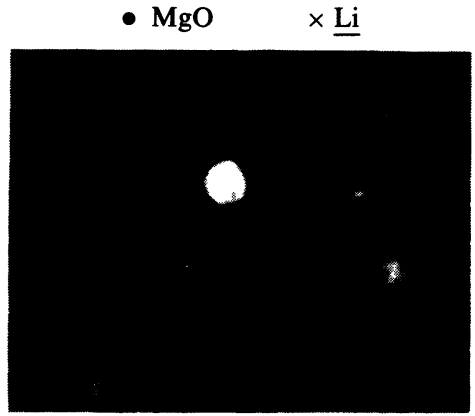

Fig. 3. - MgO implanté avec des ions $\mathrm{Li}^{+}$de $250 \mathrm{keV}$ $\left(10^{17}\right.$ ions. $\left.\mathrm{cm}^{-2}\right)$ et recuit à $1173 \mathrm{~K}$ : diagramme de diffraction et image du précipité (champ sombre avec $(\overline{200}){ }^{*} \mathrm{Li}$ ).

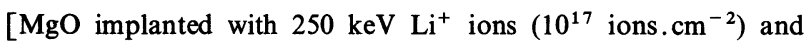
annealed at $1173 \mathrm{~K}$ : diffraction pattern and precipitate micrography (dark field with $\left.(\overline{2} 00){ }^{*} \mathrm{Li}\right)$.]

franges sont perpendiculaires à $\mathbf{g}$ (moiré parallèle), visibles en champ clair et en champ sombre. Lesmicrographies de la figure 4 , effectuées avec des $\mathrm{g}$ différents, montrent que les mêmes précipités don- nent toujours des franges de moiré. Des franges du même type, mais d'espacement différent, sont aussi obtenues pour $\mathrm{g}$ de type (200).

La présence des franges de moiré pour différents vecteurs $g$ suggère que ces précipités ont la même structure (cubique à faces centrées) que le cristal $\mathrm{MgO}$. Ces précipités sont en épitaxie avec la matrice et leur orientation par rapport à celle-ci est la suivante :

$$
\text { (001) } \mathrm{Li} / /(001) \mathrm{MgO} \text { et }[100] \mathrm{Li} / /[100] \mathrm{MgO} \text {. }
$$

Cette structure inhabituelle du lithium a déjà été observée par C. S. Barrett pour Li comprimé à basse température [7] et par M. Lambert [8] dans LiF irradié aux neutrons.

2.3.2 Cas du sodium. - Le diagramme de diffraction présente, dans ce cas, des taches supplémentaires (Fig. 2a). Par exemple, sur une coupe $(001)^{*}$ du réseau réciproque de $\mathrm{MgO}$ apparaissent des taches de diffraction au centre du réseau carré. Un champ sombre effectué avec deux de ces taches supplémentaires (Fig. $2 c$ et $d$ ) montre que ce sont les mêmes précipités qui donnent les taches de diffraction.

Le sodium a une structure cubique centrée et un paramètre $a=4,290 \AA$ très voisin de celui de $\mathrm{MgO}$ $(4,213 \AA)$. Les taches supplémentaires, situées aux centres des carrés (Fig. 2a), sont celles correspondant aux centres des faces du réseau réciproque du sodium : elles sont de type (110). Les taches très intenses sont communes à la fois à $\mathrm{MgO}$ et au sodium. Les précipités de sodium ont sensiblement le même paramètre que celui de la matrice. En effet, les constantes élastiques du sodium sont faibles par rapport à celles de la
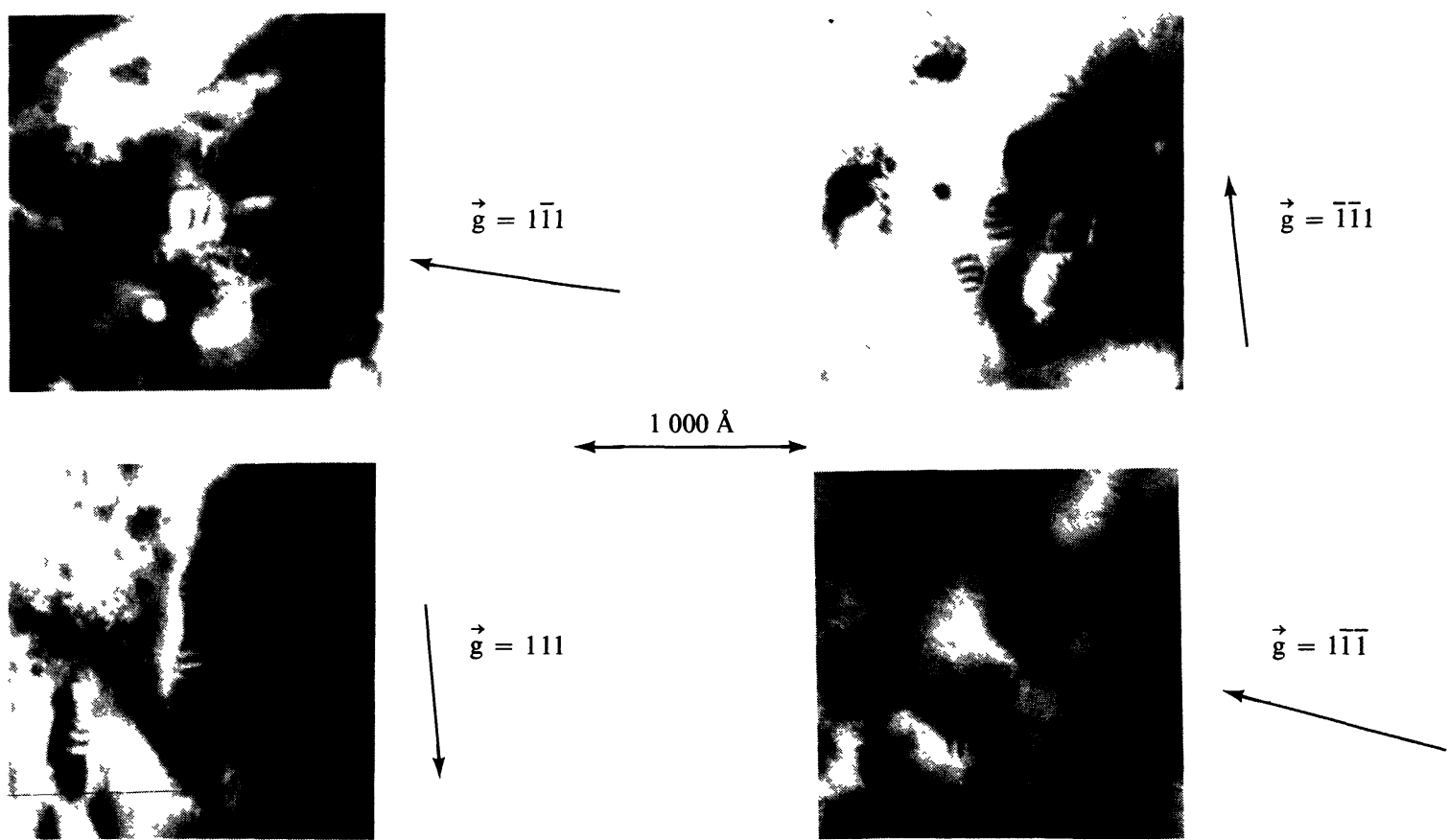

Fig. 4. - MgO implanté avec des ions $\mathrm{Li}^{+}$de $60 \mathrm{keV}$ $\left(10^{17}\right.$ ions. $\left.\mathrm{cm}^{-2}\right)$ et recuit à $1073 \mathrm{~K}:$ franges de moiré (champ sombre) sur des précipités de Li.
[MgO implanted with $60 \mathrm{keV} \mathrm{Li}^{+}$ions $\left(10^{17}\right.$ ions. $\left.\mathrm{cm}^{-2}\right)$ and annealed at $1073 \mathrm{~K}$ : moiré fringes (dark field) on Li precipitates.] 
matrice et l'accommodation entre les deux réseaux se fait aux dépens du sodium. Ces précipités sont en épitaxie avec la matrice :

$(001) \mathrm{Na} / /(001) \mathrm{MgO}$ et $[100] \mathrm{Na} / /[100] \mathrm{MgO}$.

3. Discussion. - Les observations par microscopie électronique à transmission de cristaux d'oxyde de magnésium implantés avec des ions alcalins $\left(\mathrm{Li}^{+}\right.$, $\mathrm{Na}^{+}$) indiquent que l'orientation des précipités de particules implantées par rapport à la matrice est fortement influencée par cette dernière. Lorsque le paramètre cristallin du métal alcalin est très voisin de celui de la matrice

$$
(a(\mathrm{Na})=4,290 \AA, \quad a(\mathrm{MgO})=4,213 \AA),
$$

bien que la structure soit différente, l'épitaxie s'effectue simplement :

et

$$
\text { (001) } \mathrm{Na} / /(001) \mathrm{MgO}
$$

[100] $\mathrm{Na} / /[100] \mathrm{MgO}$.

Les précipités sont alors parfaitement cohérents avec la matrice.

Par contre, lorsque les paramètres sont très différents $(a(K)=5,34 \AA)$, la relation d'orientation est compliquée, cependant il existe une influence épitaxique [1]. Les précipités sont alors semi-cohérents ou incohérents.

Dans le cas du lithium, les précipités, en début de croissance, présentent une structure inhabituelle cubique à faces centrées. Ils sont en épitaxie simple avec la matrice :

et

$$
\text { (001) } \mathrm{Li} / /(001) \mathrm{MgO}
$$

$$
\text { [100] } \mathrm{Li} / /[100] \mathrm{MgO} \text {. }
$$

A. Julg et al. [9] indiquent que la structure stable du lithium est cubique centrée, pour des amas de plus de $10^{6}$ atomes (correspondant à des précipités sphériques de diamètre supérieur à $340 \AA$ ). Pour des amas de lithium comportant de $10^{4}$ (diamètre $=74 \AA$ ) à $10^{6}$ atomes, les structures cubique à faces centrées et cubique centrée sont d'énergies comparables. Dans ces conditions, la nucléation au sein d'un matériau cubique à faces centrées $(\mathrm{MgO})$, dont le paramètre ( $a=4,213 \AA$ ) est très voisin de celui du lithium cubique à faces centrées (paramètre déterminé par C. S. Barrett [7] = 4,41 $\AA$ ), favorise la croissance des précipités dans cette structure. Ils sont alors parfaitement cohérents avec la matrice.

Nous avons vu que, lorsque la température de recuit augmente, les précipités grossissent. De plus, lorsque nous effectuons un reçuit, la phase liquide est obtenue puisque la température de fusion du lithium est de $180,5^{\circ}$. Ainsi, lorsque les précipités atteignent une certaine taille, ils recristallisent dans la structure cubique centrée et changent d'orientation pour abaisser leur énergie totale. Ils deviennent alors incohérents ou partiellement cohérents.

\section{Bibliographie}

[1] Treilleux, M., Thevenard, P., Chassagne, G. and Hobbs, L. W., Phys. Status Solidi (a) 48 (1978) 425.

[2] Thevenard, P., J. Physique Colloq. 37 (1976) C7-526.

[3] Marichy, G., Thèse Docteur-Ingénieur Lyon (1978).

[4] Edmonson, B. and Williamson, G. K., Philos. Mag. 9 (1964) 277.

[5] Howitr, D. G., Barnard, R. S., Hobbs, L. W. and MitCHELL, T. E., Defects in Insulating Crystals (1977) 187.
[6] Groves, G. W. and Kelly, A., Philos. Mag. 8 (1963) 1437.

[7] Barrett, C. S., Phase transformations in solids (Wiley and Sons, New York) 1951, p. 351.

[8] Lambert, M., Mazieres, Ch. and Guinier, A., J. Phys. Chem. Solids 18 (1961) 129.

[9] Julg, A., Benard, M., Bourg, M., Gillet, M. and Gillet, E. Phys. Rev. B 9 (1974) 3248. 